\title{
The Association between mrna Expression Levels of Ephx1 and Prognosis of Acute Myeloid Leukemia
}

\author{
ChongMei Huang\#, Hui Cheng\#, XiaoQian Xu, XianMin Song, Li Chen*and JianMin Yang*
}

Institute of Hematology, Changhai Hospital, Second Military Medical University, Shanghai 200433, PR China \#Contributed equally to this work

\begin{abstract}
The human epoxide hydrolase 1 (EPHX1) is a metabolism gene, which is responsible for the first phase of the xenobiotic metabolism. The product, microsomal epoxide hydrolase $(m H E)$, plays an important role in the detoxification of carcinogenic polycyclic aromatic hydrocarbons. Many reports also have studied the correlation between EPHX1 gene polymorphisms and development and generation of cancers. However, few of studies on $E P H X 1$ participating in chemotherapeutics metabolism were reported. The purpose of the present study was to evaluate the prognostic significance of the EPHX1gene expression in acute myeloid leukemia (AML). Using real time-quantitative polymerase chain reaction (RT-qPCR), mRNA expression levels of EPHX1gene was measured in bone marrow samples of newly diagnosed AML patients $(n=46)$ and control group $(n=12)$ without tumors. The levels expression of EPHX1 had a significant association with the treatment response and prognosis of $A M L$ patients. Additionally, the EPHX1 expression levels can aid for an improved understanding of the multidrug resistant mechanism in AML.
\end{abstract}

Keywords: Epoxide hydrolase1; mRNA expression levels; Acute myeloid leukemia; Prognosis

\section{Introduction}

Acute myeloid leukemia (AML) originates from accumulation of abnormal blasts in the marrow. Diagnosis rests on demonstration that the marrow or blood has $>20 \%$ blasts with specifical surface markers CD33 and CD13 [1]. Approximately 10\% of AML are classified as having core binding factor $(\mathrm{CBF})$ leukemia with a heterodimeric transcription factor complex comprising RUNX1(AML1, CBFa) and $\mathrm{CBF} \beta$, which plays a critical role in hematopoiesis [2]. The standard therapy for AML over the past 30 years has been centered on the traditional " $7+3$ " regimen consisting of daunorubicin, administer over 3 days alongside cytarabine, administered over 7 days [1]. With " $7+3$ " induction, complete remission (CR) rates of $40-80 \%$ have been reported [3]. In recent years, in order to improve the curative effect, people tried to increase the dose of chemotherapy, which significantly increased treatment-related deaths, CR rates but no significant improvement, relapse rates reached up to $53-60 \%$ after CR, and survival rates for 5 years was merely $22 \%$ [4]. Hence, multidrug resistance to chemotherapy considerably reduces the rate of treatment success and increases the risk of relapse.

The human epoxide hydrolase 1 (EPHX1) gene is $35.48 \mathrm{~kb}$ with nine exons and eight introns on chromosome 1q42.1, which plays a critical role in both the activation and the detoxification of polycyclic aromatic hydrocarbons (PAHs) and aromatic amines [5,6]. The product, microsomal epoxide hydrolases (mHEs), are a family of enzymes responsible in the first phase of the xenobiotic metabolism [7]. The enzymes can potentially either deactivate or activate carcinogenic compounds [8]. As we all know most of chemotherapeutics which are used for cancer patients almostly based on PAHs, especially leukemia. A few of leukemia patients are unsusceptibility for chemotherapy in clinic, which can be relative to chemotherapeutics metabolism. The present study is a novel investigation on EPHX1 that examines the mRNA expression levels in AML and evaluates its association with the response to therapy in these patients.

\section{Materials and Methods}

\section{Patients and sample preparation}

A retrospective analysis of 46 consecutive patients undergoing intensive induction chemotherapy formal at ChangHai Hospital between January 2010 and August 2013 was conducted. Patients received induction with either " $7+3$ " or a similar regimen with daunorubicin and cytarabine-based induction and consolidation therapies. Samples obtained fully informed consent from all the parents. 46 bone marrow samples from de novo AML patients were studied, including $\mathrm{CBF}^{+}(\mathrm{n}=12)$ and $\mathrm{CBF}^{-}(\mathrm{n}=34)$, and compared to 12 bone marrow samples without tumors. Mononuclear cell isolation was performed using Lymphoprep (Hao-Yang Biological Manufacture, Ltd, Tian Jin, China).

\section{mRNA extraction and assessment}

The expression levels of EPHX1 was detected via RT-qPCR method based on SYBR. Total RNA extraction from mononuclear cells was carried out using Trizol Reagent (Invitrogen, Carlsbad, CA, USA). The amount and quality of extracted RNA was measured by a BioPhotometer (Biotek, Hamburg, USA). Subsequently, $1 \mu \mathrm{g}$ RNA was converted to cDNA using a cDNA synthesis kit (Takara Bio, Inc., Tokyo, Japan). The housekeeping gene, glyceraldehyde 3-phosphate dehydrogenase $(G A P D H)$, was selected as an internal control. Specific primers were as

*Corresponding authors: Li Chen, Department of Hematology, Changhai Hospital Shanghai 200433, PR China, Tel: 86-21-3116-1292; E-mail: yuhe0628@163.com

JianMin Yang, Department of Hematology, Changhai Hospital, Shanghai 200433 PR China, Tel: 86-21-3116-1288; E-mail: JianMin Yang @medmail.com.cn

Received November 19, 2015; Accepted November 29, 2015; Published December 13, 2015

Citation: Huang C, Cheng H, Xu X, Song X, Chen L, et al. (2015) The Association between mrna Expression Levels of Ephx1 and Prognosis of Acute Myeloid Leukemia. J Integr Oncol 4: 154. doi:10.4172/2329-6771.1000154

Copyright: $\odot 2015$ Huang C, et al. This is an open-access article distributed unde the terms of the Creative Commons Attribution License, which permits unrestricted use, distribution, and reproduction in any medium, provided the original author and source are credited. 
Citation: Huang C, Cheng H, Xu X, Song X, Chen L, et al. (2015) The Association between mrna Expression Levels of Ephx1 and Prognosis of Acute Myeloid Leukemia. J Integr Oncol 4: 154. doi:10.4172/2329-6771.1000154

follows: EPHX1-F: 5'-CTCCTCACTTCAGTGCTGGG-3', EPHX1-R: 5'-GAGGATGCCTCTGAGAAGCC-3', Product Size: 569bp, and GAPDH-F: 5'-GATTTGGTCGTATTGGGCGC-3', GAPDH-R: 5'AGTGATGGCATGGACTGTGG-3', Product Size: 518bp. SYBR Premix Ex Taq II kit (Takara Bio, Inc., Tokyo, Japan) was used for RT-qPCR that was carried out through an optimized program (30 sec pre-incubation at $95^{\circ} \mathrm{C}, 5 \mathrm{sec}$ denaturation at $95^{\circ} \mathrm{C}, 34 \mathrm{sec}$ annealing at $60^{\circ} \mathrm{C}$ in 40 cycles) on the ABI 7500 (Applied Biosystems, Foster City, CA, USA).

\section{Evaluation of treatment response}

Newly diagnosed AML patients were treated mainly based on version.2.2010 AML NCCN clinical practice guideline. Treatment response was assessed according to morphology, multiparameter flow cytometry and RT-qPCR in the year following the initiation of the treatment. Persistence high expression of fusion gene or relapse after complete remission were considered as a poor response to therapy and resistance.

\section{Statistical analysis}

Graphpad Prism 5 software was used for statistical analysis of present study. Correlations between gene expression levels and AML prognostic factors were measured using Log-rank (Mantel-Cox) Test. Associations with gene expression among different groups were performed using $t$-test. Data are shown as mean \pm standard error of the mean (SEM) and $\mathrm{P}<0.05$ was considered to indicate a statistically significant difference.

\section{Results}

\section{Patients presentation}

Amount to 46 de novo AML patients were involved in the study. 12 of the total patients with single $\mathrm{CBF}$ fusion gene positive, belonged to better group. Three patients succumbed during the induction stages due to serious bleeding and infection. Nine patients, who relapsed during the first year of treatment, were considered as treatment resistant and refractory patients, and subsequently seven of relapse patients were processed to allogeneic hematologic stem cell transplantation.

\section{mRNA expression levels of the EPHX1 gene}

The mRNA expression levels of EPHX1 were significantly different between de novo AML patients and the control group (mean \pm SEM, $0.5873 \pm 1.2975 \mathrm{vs} 0.0365 \pm 0.0152$, respectively; $\mathrm{P}=0.0376$ ) (Figure 1). The $E P H X 1$ gene expression levels shown a significant difference between the $\mathrm{CBF}^{-}$group and control group, $\mathrm{CBF}^{+}$group, and had no difference between $\mathrm{CBF}^{+}$group and control group (Table 1 and Figure 2).

\section{Expression levels of mHE protein}

Western blotting was used for the mononuclear cell of 4 persistence non-remission patients and 2 controls without tumors. The product of $E P H X 1$ gene, $m H E$, which shown obvious difference (Figure 3).

\section{The EPHX1 gene expression and prognosis}

In this study, 46 AML patients were divided into two groups according to media value of the EPHX1 gene expression, $\leq 0.07(\mathrm{n}=24)$ group and $>0.07(\mathrm{n}=22)$ group. Possible prognostic difference, including over survival (OS), relapse rates (RR), disease-free survival (DFS) and event-free survival (EFS), were investigated between two groups. Logrank (Mantel-Cox) Test demonstrated a significant difference on OS $(\mathrm{P}=0.0382), \mathrm{RR}(\mathrm{P}=0.0216)$ and $\mathrm{DFS}(\mathrm{P}=0.0449)$, and had no significant difference on EFS ( $\mathrm{P}=0.0621)$ (Figure 4).

\section{Discussion}

The present study identifies the role of EPHX1 gene expression as a prognostic factor in AML for the first time. In addition, the data showed

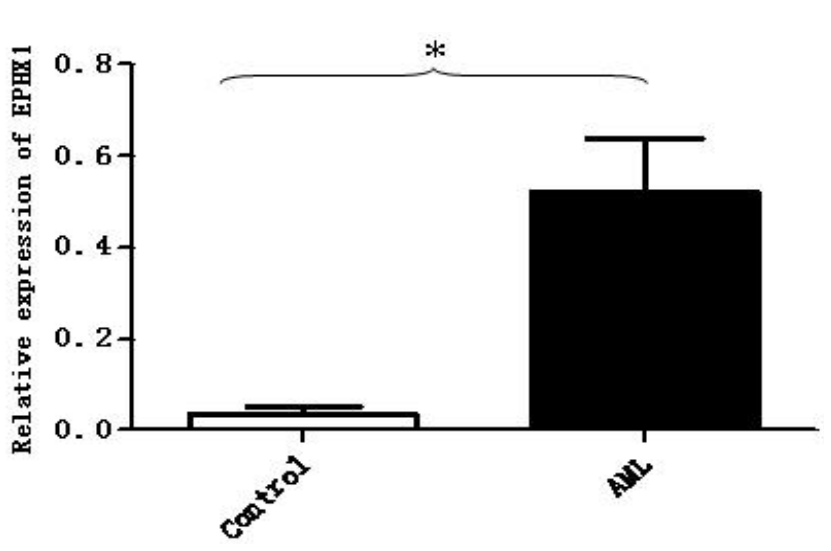

Figure 1: Relative mRNA expression levels of EPHX1 between control group and acute myeloid leukemia group. ${ }^{*} \mathrm{P}<0.05$.

\begin{tabular}{|c|c|c|c|c|}
\hline \multicolumn{4}{|c|}{ EPHX1 mRNA expression levels (mean \pm SEM) } & \multirow[t]{2}{*}{ P-value } \\
\hline Control $(n=12)$ & $\mathrm{CBF}$ & $=12)$ & $\operatorname{CBF}(n=34)$ & \\
\hline $0.0365 \pm 0.0152$ & 0.1519 & \pm 0.0665 & - & 0.1051 \\
\hline - & 0.1519 & \pm 0.0665 & $0.6829 \pm 0.1508$ & 0.0461 \\
\hline $0.0365 \pm 0.0152$ & - & - & $0.6829 \pm 0.1508$ & 0.0476 \\
\hline
\end{tabular}

Table 1: Correlation of EPHX1 gene expression levels among control group, CBF+ group and CBF-group.

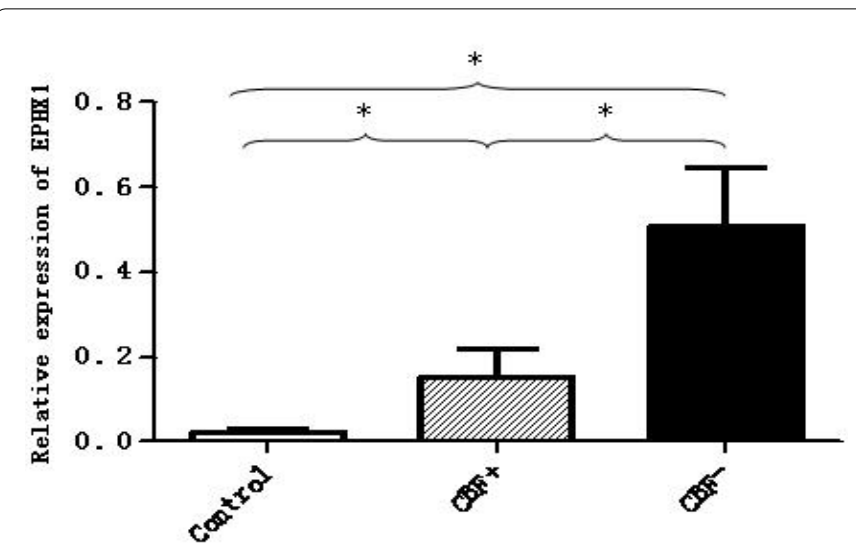

Figure 2: Correlation of EPHX1 gene expression levels between contro group and AML subgroups.

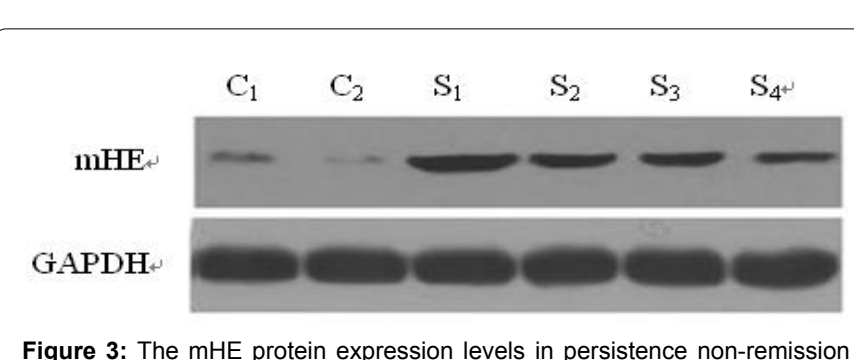
AML patients are significantly higher than control group. 

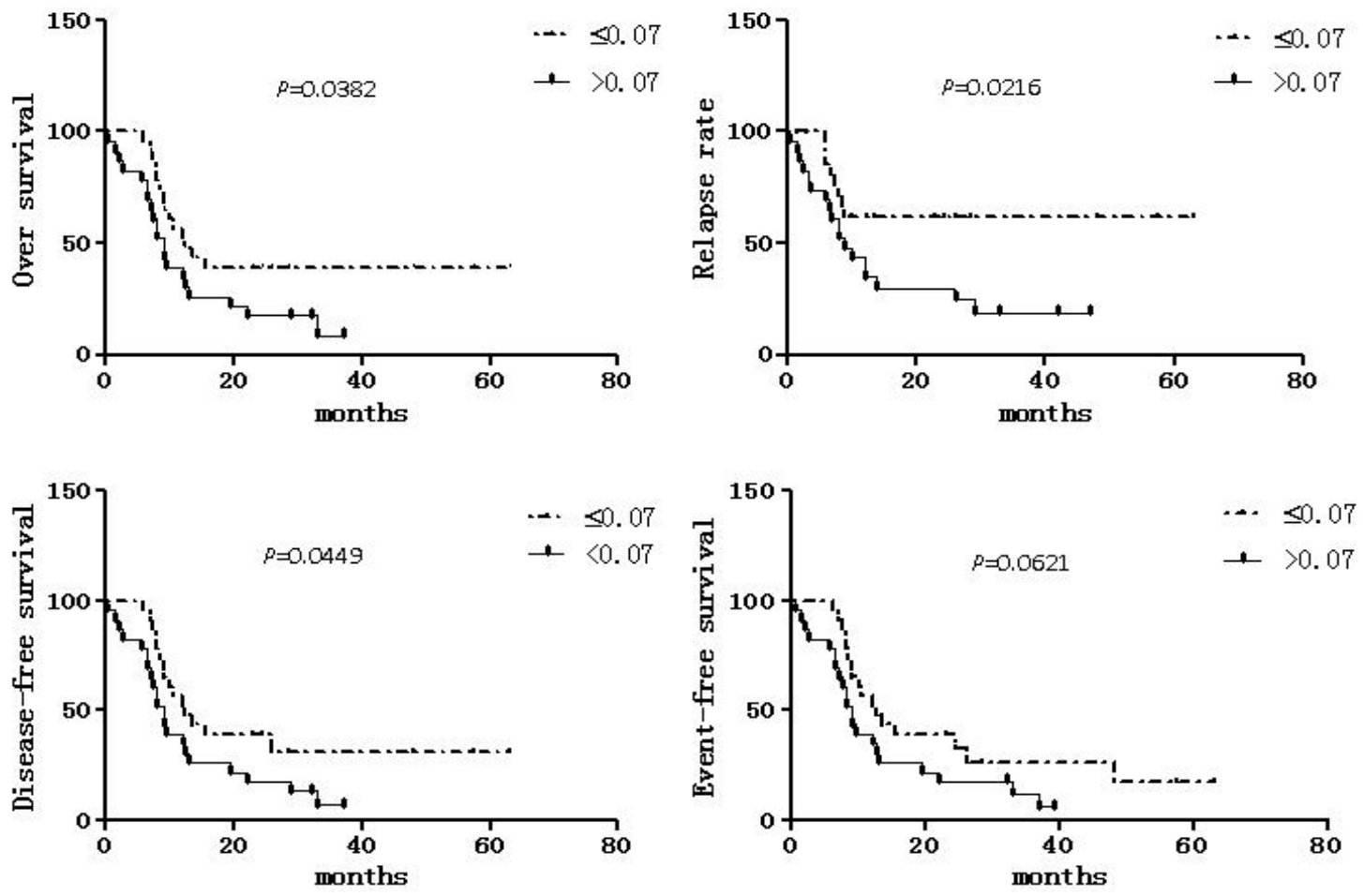

Figure 4: Association between EPHX1 gene expression levels and prognosis. Log-rank (Mantel-Cox) Test demonstrated a significant difference.

that EPHX1 gene expression in de novo patients is much higher than control group $(\mathrm{P}=0.0376)$. However, what many reports shown was that the EPHX1 gene polymorphisms play an important role in both the activation and detoxification of carcinogenic PAHs and aromatic amines [9-11]. Chemotherapeutics (daunorubicin, cytarabine, et.al) which are used for cancer patients almostly based on PAHs, which are also carcinogens in essence. Parts of AML patients are unsusceptibility for chemotherapy in clinic, which can be relative to the EPHX1 gene participating in chemotherapeutics metabolism. The present results indicate a significant increase in mRNA expression levels of EPHX1 in $\mathrm{CBF}^{-}$patients compared to $\mathrm{CBF}^{+}$patients and the control group. These data suggest that $E P H X 1$ has an adverse impact on response to therapy. Therefore, increased expression of EPHX1 can be a poor prognostic factor for AML. Few studies on the association between EPHX1 gene expression and prognosis were reported in AML patients. Up to now only few reports shown EPHX1 gene polymorphisms in xenobiotic metabolizing genes are associated with altered metabolism of carcinogens in acute leukemia [12]. This study indicates an association between the increased mRNA expression levels of EPHX1 and poor treatment response and early drug resistance.

The product of $E P H X 1$ gene, $m E H$, is a drug metabolizing enzyme found on the endoplasmic reticulum of many tissues and is responsible for catalyzing the hydration of reactive epoxide intermediates that are formed bycytochrome P450s, including PAHs [10,13]. The results shown $m E H$ protein was high expression in persistence non-remission patients compared to control group. By contrast, it is suggested that EPHX1 may have an impact on multidrug resistance in AML. The present study has examined, for the first time, the correlation between EPHX1gene expression and prognosis in AML. Statistical tests were performed to investigate the association between EPHX1 mRNA expression levels and clinical prognosis, including OS, RR, DFS and
EFS. The results indicated high EPHX1 expression mRNA levels group had a significant difference compared to low expression group on OS, RR, DFS, and had no significant difference on EFS. Additionally, this study may open up more opportunities to understand the multidrug resistance of AML.

\section{Conclusion}

From above, the data suggestions EPHX1 mRNA overexpression can be proposed as a negative prognostic factor in AML, which increases the risk of resistance to chemotherapy. It is suggested that EPHX1 may be associated with detoxification of chemotherapeutics. The results shown that overexpression of EPHX1 may apply its adverse effect on response to treatment. In conclusion, the exact function of EPHX1 in AML for increasing the risk of resistance to therapy remain to be explored.

\section{Acknowledgements and Declaration of Interests}

\section{Authors' contributions}

$\mathrm{CH}$ and $\mathrm{HC}$ contributed equally to this work. $\mathrm{CH}$ detected samples, collected, analyzed and interpreted data, and $\mathrm{HC}$ wrote the manuscript; LC critically reviewed the manuscript and importantly given her advice in English; $X X, X S$, and JY performed diagnosis and treatment for the patient.

\section{Funding supports}

The work was supported with the grants from National Natural Science Foundation of China $(81400133,30973495)$, and the Shanghai Science and Technology Committee (15ZR1412600 to L.C).

\section{Competing interests}

The authors declare that no potential conflicts of interest relevant to this article were reported.

\section{References}

1. Reagan JL, Sullivan MR, Winer ES, Lansigan F, Cardin MS, et al. (2015) 
Citation: Huang C, Cheng H, Xu X, Song X, Chen L, et al. (2015) The Association between mrna Expression Levels of Ephx1 and Prognosis of Acute Myeloid Leukemia. J Integr Oncol 4: 154. doi:10.4172/2329-6771.1000154

Potential for improved survival with intensification of daunorubicin based induction chemotherapy in acute myeloid leukemia patients who do not receive transplant: A multicenter retrospective study. Leuk Res 39: 812-817.

2. Goyama S, Mulloy JC (2011) Molecular pathogenesis of core binding factor leukemia: current knowledge and future prospects. Int J Hematol 94: 126-133.

3. Lee JH, Joo YD, Kim H, Bae SH, Kim MK, et al. (2011) A randomized trial comparing standard versus high-dose daunorubicin induction in patients with acute myeloid leukemia. Blood 118: 3832-3841.

4. Roboz GJ (2012) Current treatment of acute myeloid leukemia. Curr Opin Oncol 24: 711-719.

5. Puranik YG, Birnbaum AK, Marino SE, Ahmed G, Cloyd JC, et al. (2013) Association of carbamazepine major metabolism and transport pathway gene polymorphisms and pharmacokinetics in patients with epilepsy. Pharmacogenomics 14: 35-45.

6. Wang Q, Pang W, Cui Z, Shi J, Liu Y, et al. (2013) Upregulation of soluble epoxide hydrolase in proximal tubular cells mediated proteinuria-induced renal damage. Am J Physiol Renal Physiol 304: F168-176.

7. Ginsberg G, Guyton K, Johns D, Schimek J, Angle K, et al. (2010) Genetic polymorphism in metabolism and host defense enzymes: implications for human health risk assessment. Crit Rev Toxicol 40: 575-619.

8. Abdel-Rahman SZ, Ammenheuser MM, Ward JB Jr (2001) Human sensitivity to 1,3-butadiene: role of microsomal epoxide hydrolase polymorphisms. Carcinogenesis 22: 415-423.

9. Zhao ZQ, Guan QK, Yang FY, Zhao P, Zhou B, et al. (2012) System review and metaanalysis of the relationships between five metabolic gene polymorphisms and colorectal adenoma risk. Tumour Biol 33: 523-535.

10. Arand M, Cronin A, Adamska M, Oesch F (2005) Epoxide hydrolases: structure function, mechanism, and assay. Methods Enzymol 400: 569-588.

11. Tumer TB, Sahin G, Arinc E (2012) Association between polymorphisms of EPHX1 and XRCC1 genes andthe risk of childhood acute lymphoblastic leukemia. Arch Toxicol 86: 431-439.

12. Chauhan PS, Ihsan R, Mishra AK, Yadav DS, Saluja S, et al. (2012) High order interactions of xenobiotic metabolizing genes and $\mathrm{P} 53$ codon 72 polymorphisms in acute leukemia. Environ Mol Mutagen 53: 619-630.

13. Duan H, Yoshimura K, Kobayashi N, Sugiyama K, Sawada J, et al. (2012) Development of monoclonal antibodies to human microsomal epoxide hydrolase and analysis of "preneoplastic antigen"-like molecules. Toxicol Appl Pharmacol 260: 17-26. 